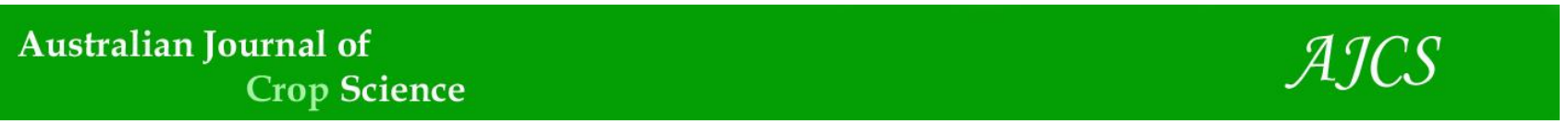

AJCS 14(08):1319-1327 (2020)

ISSN:1835-2707

doi: 10.21475/ajcs.20.14.08.p2591

\title{
Fuzzy logic simulation of oat yield after using hydrogel and nitrogen biopolymer management
}

\author{
Osmar Bruneslau Scremin ${ }^{1}$, José Antonio Gonzalez da Silva ${ }^{1}$, Ivan Ricardo Carvalho* ${ }^{1}$, Ângela Teresinha \\ Woschinski De Mamann ${ }^{3}$, Odenis Alessi ${ }^{1}$, Vanessa Pansera ${ }^{1}$, Francine Lautenchleger ${ }^{2}$, Roberto \\ Carbonera ${ }^{1}$, Leonardo Norbert ${ }^{1}$, Natiane Carolina Ferrari Basso ${ }^{1}$
}

\author{
${ }^{1}$ Regional University of the Northwest of Rio Grande do Sul, ljuí, Brazil. Street of Comércio 3000, \\ Universitário, ljuí/RS, Brazil \\ ${ }^{2}$ State University of Central-West - Street Presidente Zacarias, 875, Guarapuava/PR, Brazil \\ ${ }^{3}$ Federal Institute of Education, Science and Technology of Rio Grande do Sul, Street Nelsi Ribas Fritsch, \\ 1111, Neighborhood Esperança, Ibirubá/RS, Brazil
}

\begin{abstract}
*Corresponding author: carvalho.irc@gmail.com
Abstract

The fuzzy logic is an efficient tool for simulation and validation of new technologies in agriculture. The objective of the study is to adapt the fuzzy logic model for simulation of biomass and oat grain yield by nitrogen involving the nonlinearity of the maximum air temperature in the conditions of use of the biopolymer hydrogel, considering high succession systems and low release of residual $\mathrm{N}$. The study was conducted in 2014 and 2015, in a randomized block design with four replicates in a $5 \times 5$ factorial. Five hydrogel doses $\left(0,30,60,90\right.$ and $\left.120 \mathrm{~kg} \mathrm{ha}^{-1}\right)$ were added in the groove next to the seed; and 5 doses of $\mathrm{N}$-fertilizer $(0,30,60,90$ and $120 \mathrm{~kg}$ $\mathrm{ha}^{-1}$ ) applied at the fourth expanded leaf stage, respectively. The cultivar was URS Corona. The pertinence functions and the linguistic values established in the input and output variables to simulate the biomass yield and oat grains in the succession systems are adequate observed productivity. The fuzzy model makes it possible to estimate the biomass and oat grains productivity efficiently under the conditions of use of the hydrogel as a function of the nitrogen doses and maximum air temperature, adding to the existing models of simulation.
\end{abstract}

Keywords: Avena sativa, maximum air temperature, innovation, diffuse logic, sustainability.

Abbreviations: BY_biological yield; GY_grain yield; N_nitrogen; $T_{M A ́ x}$ maximum air temperature.

\section{Introduction}

In agriculture, mathematical modeling techniques are based on processes involving multiple factors. Due to this condition, it is one of the growing areas in research that seeks to improve the understanding of environmental and management conditions in agricultural crops (Leal et al., 2015; Brezolin et al., 2017). In this perspective, the phenomena of nature together with biological processes, because they represent data that behave in an imprecise way, need efficient models that facilitate the understanding and the possibility of simulations (Mota et al., 2007; Schiassi et al., 2015). The fuzzy models are techniques that allow the description of complex systems of nonlinear behaviors, produced from rules, that must be elaborated by specialists, providing their experience to the elaboration of an "If $<$ condition>Then<result> " system (De et al. 2009; Silva et al., 2014).

In recent years, oat harvesting in southern Brazil has become an important alternative in winter cropping systems, due to its high acceptance in the food industry (Crestani et al., 2014; Arenhardt et al., 2017). However, because it is a grass, it is highly nitrogen-dependent to reach higher yields with grain quality required by the industry (Silva et al., 2016; Marolli et al., 2017). It is worth noting that nitrogen management shows great complexity on the environment, and can be leached in rainy years and volatilized in high temperature conditions, generating nutrient losses, consequently increasing production costs and environmental contamination ambiental (Mantai et al., 2015; Silva et al., 2016). The maximum efficiency of nutrient utilization by the plant is strongly dependent on adequate soil moisture (Acosta et al., 2014; Marolli et al., 2018). Therefore, under actual growing conditions, the nitrogen fertilization moment does not always coincide with adequate soil moisture and air temperature conditions (Arenhardt et al., 2015; Ward et al., 2018). In this context, the use of water hydro-retentive added to the soil can favor the maintenance of moisture and improve the efficiency of nitrogen use with reduced nutrient losses (Oliveira, et al., 2004; Reza et al., 2011). Hydrogels are biodegradable threedimensional polymer networks, which retain water in their structure, swell, forming a gel, capable of hydrating and releasing water for a long period of time (Sartore et al., 2013; Guilherme et al., 2015).

The use of fuzzy logic may represent a potential tool for the simulation of biological processes involving non-linear 
relationships of environmental conditions with cultivated agroecosystems. In addition, in the validation and optimization of new management technologies aimed at greater sustainability of yield in agriculture, such as the innovative use of technology of hydro-retentors in grainproducing crops. In this context, the objective of the study is to adapt the fuzzy logic model to the simulation of biomass and oat grain yield by nitrogen involving the non-linearity of the maximum air temperature in the conditions of use of the biopolymer hydrogel, considering high and reduced residual $\mathrm{N}$ release.

\section{Results and discussion}

\section{Meteorological conditions}

In Figure 1, in the oat crop cycle temperatures were higher in 2014 than in 2015. In addition, higher precipitation values were also observed in 2014 compared to 2015. In 2014, accumulated precipitation was of $952 \mathrm{~mm}$ (Figure 1A) and in 2015 of $817 \mathrm{~mm}$ (Figure 1B). These volumes are close to the historical average of the last 20 years $(900 \mathrm{~mm})$, but with different precipitation distribution between the years of cultivation. In 2014, lower rainfall periods occurred at the beginning of the crop cycle and higher peak temperatures over the cycle. These conditions favor the losses of nitrogen by volatilization and reduce the stimuli to the production of new tillers, a component directly linked to grain yield. From mid-cycle to near-maturation (2014), rainfall volumes were more expressive, which favored longer periods of less sunshine, consequently reducing the efficiency of photosynthesis. In the year 2015 , the highest volume of rainfall was from the emergence until close to 35 days of oat development, and with maximum temperatures lower than those recorded in 2014. These conditions favor maintenance of soil moisture promoting greater efficiency of nitrogen use by the plant. From the middle of the cycle to maturation, rainfall volumes were better distributed and of lower intensity, development conditions that justify the greater yield of oat in the year 2015.

In the cropping systems, the condition of the agricultural year is the factor that contributes most to the yield variation (Neto et al., 2012; Hideo et al., 2016). In wheat and oat, the condition of agricultural year is predominantly defined by air temperature and distribution and volume of rainfall (Arenhardt et al., 2015). The temperature and precipitation interfere with the decomposition rate of the residues in contact with the soil and affect the efficiency of nitrogen use by the plant (Acosta et al., 2014). Arenhardt et al. (2015) point out that long periods of rain during the cycle reduce the efficiency of light and nutrient utilization to photosynthesis, interfering with the development, yield and quality of grains during harvest (Castro et al., 2012). The air temperature is also decisive on the yield elaboration, acting as catalyst of the biological processes, reason why the plants require a minimum and maximum temperature for normality of the physiological activities (Guarienti et al. 2004). In oat, the favorable climate is described as one of milder temperatures and of radiation quality in favor of tillering and grain filling, without occurrence of rains in great quantity and intensity, however, that favors the adequate supply of moisture stored in the soil (Castro et al., 2012; Marolli et al., 2017).

\section{Biological behavior of oat productivity}

Figures 2 and 3 show the parameters of linear $\left(y= \pm b_{1} x \pm\right.$ $\left.b_{0}\right)$ and quadratic $\left(y= \pm b_{2} x^{2} \pm b_{1} x \pm b_{0}\right)$ functions for the interpretation of the biological behavior of oat yield by nitrogen in the conditions of use of the biopolymer hydrogel. In linear functions the starting point of agronomic performance is given by the linear coefficient $\left(b_{0}\right)$ and slope of the line by the angular coefficient $\left(b_{1}\right)$ in the comparison between the real data and those estimated by the fuzzy logic. The parameter $b_{1}$ of the linear function provides the agronomic efficiency, indicating the relationship between the yield obtained per unit of nitrogen supplied. In quadratic functions, the vertex $\left(\frac{-b_{1}}{2 b_{2}}\right)$ represents the maximum use of nitrogen on the expression of yield, ie, maximum technical efficiency.

In the soybean/oat system, for grain yield (Figure 2), regardless of the condition of hydrogel use, all functions presented quadratic behavior, with the possibility of calculating the maximum nutrient utilization. In this sense, using as an example the functions of graph $\mathrm{C}$ of Figure 2, the points observed in the field and simulated by fuzzy logic were approximate, and with maximum grain yield by the technical efficiency of $76 \mathrm{~kg} \mathrm{ha}^{-1}$ of nitrogen. In the corn/oat system (Figure 2), the doses 0 and $30 \mathrm{~kg} \mathrm{ha}^{-1}$ of hydrogel presented quadratic behavior and those of 60,90 and $120 \mathrm{~kg}$ $\mathrm{ha}^{-1}$ of hydrogel indicated a linear trend. The linear behavior functions showed very approximate estimates of agronomic efficiency, with emphasis on the equations of the graphs I and $\mathrm{J}$ of Figure 2 , with an angular coefficient $\left(b_{1}\right)$ around 13 , that is, for every $1 \mathrm{~kg}$ of nitrogen supplied per hectare, there is increase of $13 \mathrm{~kg} \mathrm{ha}^{-1}$ of grain yield, either in the real condition or simulated by fuzzy logic.

In Figure 3, for biomass yield, independent of the succession system and hydrogel use condition, all functions exhibited linear behavior. The agronomic efficiency of the equations expressing the real condition and simulated by fuzzy logic were also approximate. In this perspective, the example of the functions of Graph J, such as 53 and $54 \mathrm{~kg}$ of biomass yield per hectare for each $1 \mathrm{~kg} \mathrm{ha}^{-1}$ of nitrogen supplied, in the real condition and simulated by fuzzy logic, respectively, is highlighted. Therefore, Graphs A and C show a greater distance between the angular coefficients, but within an acceptable perspective of the variability of biomass yield for each $1 \mathrm{~kg}$ of nitrogen supplied. Therefore, by providing the dimension that the linear functions of expression of biomass yield simulated in the fuzification process accompany the results obtained in the actual bio-experimental conditions. In addition, allowing inference that associate the meteorological condition with management technologies in the actual representation of cultivated biosystems.

\section{Simulation of oat productivity by Fuzzy Logic}

Tables 3 and 4 present the mean and lower and upper limits of the confidence interval of the real and simulated values by fuzzy logic to the biomass and oat grains yield in the use of hydrogel and nitrogen. In this perspective, regardless of the succession system and hydrogel dose, the $\mathrm{N}$-fertilizer doses provided a change in the biomass and grains yield. In the soybean/oat system (Table 3 ), in general, the increase of $\mathrm{N}$-fertilizer provided an increase in grain yield to the point of $90 \mathrm{~kg} \mathrm{ha}^{-1}$. Fact indicating reaching stability of nutrient utilization, regardless of hydrogel dose. On the other hand, in general, the increase of $\mathrm{N}$-fertilizer promoted greater 
Table 1. Fuzzy logic rules for simulation of oat grain yield in succession systems.

\begin{tabular}{|c|c|c|c|c|c|c|c|c|c|}
\hline \multicolumn{2}{|l|}{$\mathrm{N}$} & \multirow{2}{*}{ Year } & \multicolumn{2}{|c|}{$\mathrm{T}_{\text {MÁx }}\left({ }^{\circ} \mathrm{C}\right)$} & \multicolumn{3}{|c|}{ Output Linguistic Variables } & \multirow[b]{2}{*}{$\mathrm{GY}_{\mathrm{Hy} 90}$} & \multirow[b]{2}{*}{$\mathrm{GY}_{\mathrm{Hy} 120}$} \\
\hline$L_{V}$ & $Q_{v}$ & & $L_{V}$ & $\mathrm{Q}_{\mathrm{V}}$ & $\mathrm{GY}_{\mathrm{Hy} 0}$ & $\mathrm{GY}_{\mathrm{Hy} 30}$ & $\mathrm{GY}_{\mathrm{Hy} 60}$ & & \\
\hline \multicolumn{10}{|c|}{ soybean/oat system } \\
\hline \multirow{2}{*}{ VL } & \multirow{2}{*}{$0,15[$} & 2015 & $\mathrm{~L}$ & $<22.5$ & $\mathrm{~L}$ & $\mathrm{~L}$ & $M$ & $\mathrm{~L}$ & $\mathrm{~L}$ \\
\hline & & 2014 & $\mathrm{H}$ & $>22.5$ & $\mathrm{~L}$ & $\mathrm{~L}$ & $\mathrm{~L}$ & $\mathrm{~L}$ & $\mathrm{~L}$ \\
\hline \multirow{2}{*}{$\mathrm{L}$} & \multirow{2}{*}{$15,45[$} & 2015 & L & $<22.5$ & $\mathrm{H}$ & $\mathrm{H}$ & $\mathrm{H}$ & $\mathrm{H}$ & $\mathrm{M}$ \\
\hline & & 2014 & $\mathrm{H}$ & $>22.5$ & $M$ & M & M & $M$ & $\mathrm{~L}$ \\
\hline \multirow{2}{*}{$\mathrm{M}$} & \multirow{2}{*}{$45,75[$} & 2015 & $\mathrm{~L}$ & $<22.5$ & $\mathrm{VH}$ & VH & VH & $\mathrm{VH}$ & $\mathrm{H}$ \\
\hline & & 2014 & $\mathrm{H}$ & $>22.5$ & $\mathrm{H}$ & $\mathrm{H}$ & $\mathrm{H}$ & $\mathrm{H}$ & $\mathrm{M}$ \\
\hline \multirow{2}{*}{$\mathrm{H}$} & \multirow{2}{*}{$75,105[$} & 2015 & $\mathrm{~L}$ & $<22.5$ & VH & VH & VH & VH & VH \\
\hline & & 2014 & $\mathrm{H}$ & $>22.5$ & $\mathrm{H}$ & $\mathrm{H}$ & $\mathrm{H}$ & $\mathrm{H}$ & $M$ \\
\hline \multirow{2}{*}{ VH } & \multirow{2}{*}[105,120]{} & 2015 & $\mathrm{~L}$ & $<22.5$ & $\mathrm{H}$ & VH & $\mathrm{H}$ & $\mathrm{H}$ & $\mathrm{H}$ \\
\hline & & 2014 & $\mathrm{H}$ & $>22.5$ & $M$ & $\mathrm{H}$ & $M$ & $M$ & $\mathrm{M}$ \\
\hline \multicolumn{2}{|c|}{ Real value } & \multicolumn{3}{|c|}{ Minimum } & 1850 & 2021 & 1965 & 1905 & 1836 \\
\hline \multicolumn{2}{|c|}{$(2014+2015)$} & \multicolumn{3}{|c|}{ Maximum } & 4025 & 4286 & 4124 & 4036 & 4005 \\
\hline \multicolumn{10}{|c|}{ corn/oat system } \\
\hline \multirow{2}{*}{ VL } & \multirow{2}{*}{$0,15[$} & 2015 & $\mathrm{~L}$ & $<22.5$ & $\mathrm{~L}$ & $\mathrm{~L}$ & $\mathrm{~L}$ & $\mathrm{~L}$ & $\mathrm{~L}$ \\
\hline & & 2014 & $\mathrm{H}$ & $>22.5$ & L & $\mathrm{L}$ & L & $\mathrm{L}$ & $\mathrm{L}$ \\
\hline \multirow{2}{*}{$\mathrm{L}$} & \multirow{2}{*}{$15,45[$} & 2015 & $\mathrm{~L}$ & $<22.5$ & $\mathrm{H}$ & $\mathrm{H}$ & $M$ & $\mathrm{H}$ & $\mathrm{H}$ \\
\hline & & 2014 & $\mathrm{H}$ & $>22.5$ & $M$ & $M$ & $\mathrm{~L}$ & $M$ & $M$ \\
\hline \multirow{2}{*}{$M$} & \multirow{2}{*}{$45,75[$} & 2015 & $\mathrm{~L}$ & $<22.5$ & VH & $\mathrm{H}$ & $\mathrm{VH}$ & VH & $\mathrm{VH}$ \\
\hline & & 2014 & $\mathrm{H}$ & $>22.5$ & $\mathrm{H}$ & $\mathrm{H}$ & $\mathrm{H}$ & $M$ & $M$ \\
\hline \multirow{2}{*}{$\mathrm{H}$} & \multirow{2}{*}{$75,105[$} & 2015 & $\mathrm{~L}$ & $<22.5$ & VH & VH & VH & VH & VH \\
\hline & & 2014 & $\mathrm{H}$ & $>22.5$ & $\mathrm{H}$ & VH & $\mathrm{H}$ & $\mathrm{H}$ & $\mathrm{H}$ \\
\hline $\mathrm{VH}$ & ] & 2015 & $\mathrm{~L}$ & $<22.5$ & VH & VH & VH & VH & VH \\
\hline & {$[103,120]$} & 2014 & $\mathrm{H}$ & $>22.5$ & $\mathrm{H}$ & VH & VH & VH & VH \\
\hline Rea & ue & & רimum & & 1164 & 1159 & 1510 & 1304 & 1211 \\
\hline$(201$ & 2015) & & ximum & & 3620 & 3821 & 3724 & 3725 & 3745 \\
\hline
\end{tabular}

$\mathrm{N}=$ nitrogen $\left(\mathrm{kg} \mathrm{ha}^{-1}\right) ; \mathrm{T}_{\mathrm{MAX}}=$ maximum average temperature; $\mathrm{L}_{\mathrm{V}}=$ linguistic variables; $\mathrm{Q}_{\mathrm{V}}=$ quantitative variables; $\mathrm{GY}=$ grain yield; $\mathrm{VL}=$ very low; $\mathrm{L}=$ low; $\mathrm{M}=$ medium; $\mathrm{H}=$ high; $\mathrm{VH}=$ very high; $\mathrm{Hy}=$ hydrogel ( $\mathrm{kg} \mathrm{ha}^{-1}$ )

A
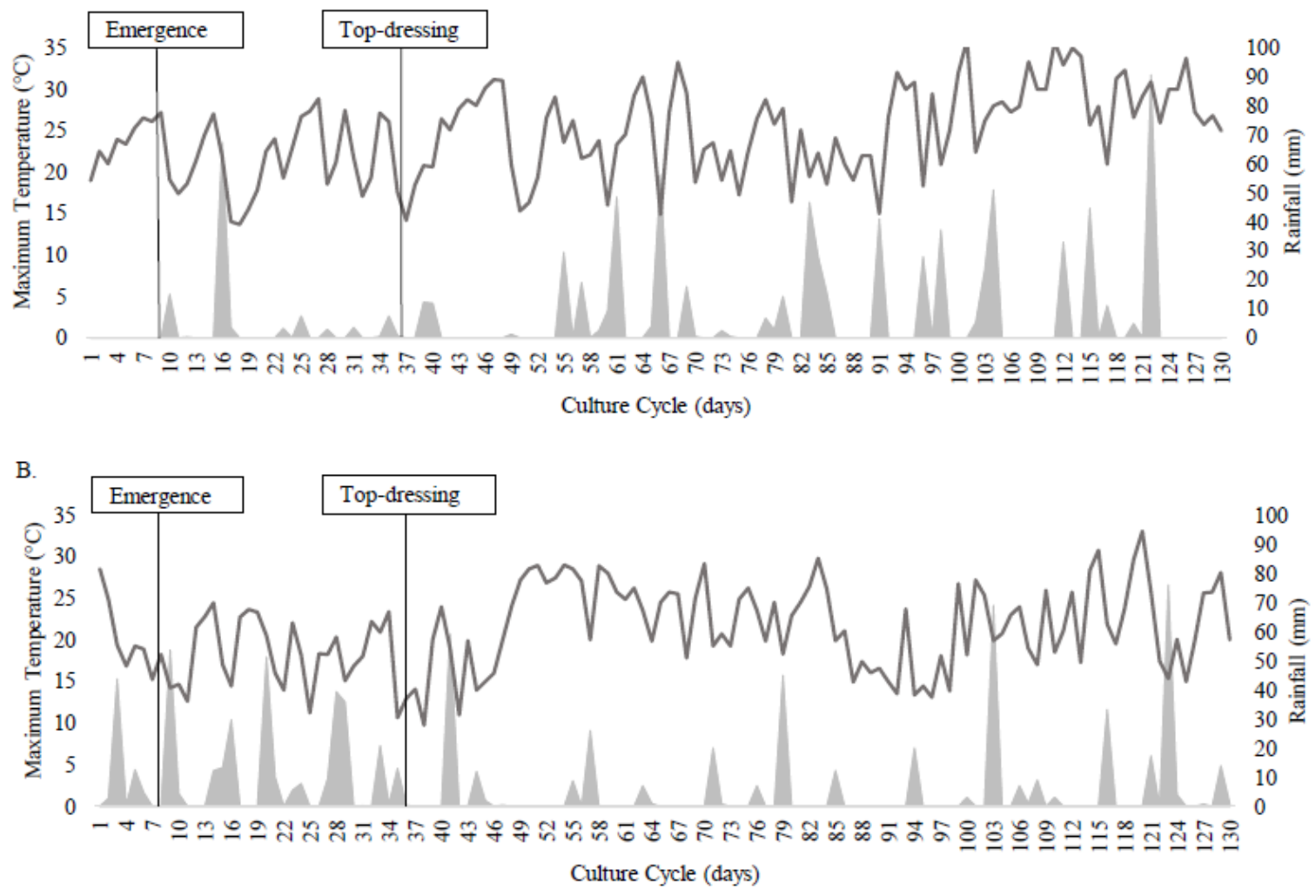

Rainfall (mm)

Maximum Temperature $\left({ }^{\circ} \mathrm{C}\right)$

Fig 1. Rainfall and maximum sowing temperature at oat harvest. (A) Agricultural year 2014; (B) Agricultural year 2015 
Table 2. Basis of fuzzy logic for the simulation of oat biomass yield in succession systems.

\begin{tabular}{|c|c|c|c|c|c|c|c|c|c|c|}
\hline \multicolumn{2}{|l|}{$\mathrm{N}$} & \multirow{2}{*}{ Year } & \multicolumn{3}{|c|}{$\mathrm{T}_{\text {MÁx }}\left({ }^{\circ} \mathrm{C}\right)$} & \multicolumn{3}{|c|}{ Output Linguistic Variables } & \multirow[b]{2}{*}{$\mathrm{BY}_{\text {Нy } 90}$} & \multirow[b]{2}{*}{$\mathrm{BY}_{\mathrm{Hy} 120}$} \\
\hline $\mathrm{L}_{\mathrm{V}}$ & $Q_{v}$ & & $\mathrm{~L}_{\mathrm{V}}$ & & $Q_{v}$ & $\mathrm{BY}_{\mathrm{Hy} 0}$ & $\mathrm{BY}_{\mathrm{Hy} 30}$ & $\mathrm{BY}_{\mathrm{Hy} 60}$ & & \\
\hline \multicolumn{11}{|c|}{ Soybean/oat system } \\
\hline \multirow{2}{*}{ VL } & \multirow{2}{*}{$0,15[$} & 2015 & $\mathrm{~L}$ & & $<22.5$ & $\mathrm{M}$ & $\mathrm{M}$ & $\mathrm{M}$ & $\mathrm{M}$ & $\mathrm{L}$ \\
\hline & & 2014 & $\mathrm{H}$ & & $>22.5$ & VL & VL & VL & VL & VL \\
\hline \multirow{2}{*}{ L } & \multirow{2}{*}{$15,45[$} & 2015 & $\mathrm{~L}$ & & $<22.5$ & $\mathrm{H}$ & $\mathrm{H}$ & $\mathrm{M}$ & $\mathrm{M}$ & $\mathrm{M}$ \\
\hline & & 2014 & & $\mathrm{H}$ & $>22.5$ & $\mathrm{~L}$ & $\mathrm{~L}$ & $\mathrm{M}$ & $M$ & M \\
\hline \multirow{2}{*}{ M } & \multirow{2}{*}{$45,75[$} & 2015 & $\mathrm{~L}$ & & $<22.5$ & $\mathrm{H}$ & VH & VH & $\mathrm{H}$ & $\mathrm{H}$ \\
\hline & & 2014 & $\mathrm{H}$ & & $>22.5$ & $\mathrm{M}$ & $\mathrm{M}$ & $M$ & $\mathrm{H}$ & $\mathrm{H}$ \\
\hline \multirow{2}{*}{$\mathrm{H}$} & \multirow{2}{*}{$75,105[$} & 2015 & $\mathrm{~L}$ & & $<22.5$ & VH & VH & VH & VH & VH \\
\hline & & 2014 & $\mathrm{H}$ & & $>22.5$ & $\mathrm{H}$ & $\mathrm{H}$ & $\mathrm{H}$ & $\mathrm{H}$ & $\mathrm{H}$ \\
\hline \multirow{2}{*}{$\mathrm{VH}$} & \multirow{2}{*}[105,120]{} & 2015 & $\mathrm{~L}$ & & $<22.5$ & VH & VH & VH & VH & VH \\
\hline & & 2014 & $\mathrm{H}$ & & $>22.5$ & $\mathrm{H}$ & VH & $\mathrm{H}$ & VH & VH \\
\hline \multirow{2}{*}{\multicolumn{2}{|c|}{$\begin{array}{l}\text { Real value } \\
(2014+2015)\end{array}$}} & \multicolumn{3}{|c|}{ Minimum } & & 4517 & 5601 & 6017 & 6782 & 7348 \\
\hline & & & num & & & 15289 & 14984 & 13361 & 14167 & 14835 \\
\hline \multicolumn{11}{|c|}{ Corn/oat system } \\
\hline \multirow{2}{*}{ VL } & \multirow{2}{*}{$0,15[$} & 2015 & $\mathrm{~L}$ & & $<22.5$ & $\mathrm{~L}$ & $\mathrm{~L}$ & $M$ & $\mathrm{M}$ & $\mathrm{L}$ \\
\hline & & 2014 & $\mathrm{H}$ & & $>22.5$ & VL & VL & $\mathrm{L}$ & VL & VL \\
\hline \multirow{2}{*}{ L } & \multirow{2}{*}{$15,45[$} & 2015 & L & & $<22.5$ & $\mathrm{H}$ & $\mathrm{H}$ & $\mathrm{H}$ & $\mathrm{M}$ & $\mathrm{M}$ \\
\hline & & 2014 & $\mathrm{H}$ & & $>22.5$ & $M$ & $\mathrm{~L}$ & VL & $\mathrm{L}$ & $\mathrm{L}$ \\
\hline \multirow{2}{*}{ M } & \multirow{2}{*}{$45,75[$} & 2015 & $\mathrm{~L}$ & & $<22.5$ & $\mathrm{H}$ & $\mathrm{H}$ & $\mathrm{H}$ & $\mathrm{H}$ & $\mathrm{H}$ \\
\hline & & 2014 & $\mathrm{H}$ & & $>22.5$ & $\mathrm{H}$ & $M$ & $M$ & $M$ & $M$ \\
\hline \multirow{2}{*}{$\mathrm{H}$} & \multirow{2}{*}{$75,105[$} & 2015 & $\mathrm{~L}$ & & $<22.5$ & VH & $\mathrm{H}$ & VH & $\mathrm{H}$ & $\mathrm{H}$ \\
\hline & & 2014 & $\mathrm{H}$ & & $>22.5$ & $\mathrm{H}$ & $\mathrm{H}$ & $\mathrm{H}$ & $\mathrm{H}$ & $\mathrm{H}$ \\
\hline \multirow{2}{*}{$\mathrm{VH}$} & \multirow{2}{*}[105,120]{} & 2015 & $\mathrm{~L}$ & & $<22.5$ & VH & VH & VH & VH & VH \\
\hline & & 2014 & $\mathrm{H}$ & & $>22.5$ & $\mathrm{VH}$ & $\mathrm{H}$ & $\mathrm{VH}$ & VH & $\mathrm{VH}$ \\
\hline Rea & lue & & hum & & & 4231 & 4122 & 5601 & 4659 & 4066 \\
\hline (20) & 2015) & & num & & & 12534 & 12132 & 12982 & 13167 & 13572 \\
\hline
\end{tabular}

$\mathrm{N}=$ nitrogen $\left(\mathrm{kg} \mathrm{ha}^{-1}\right) ; \mathrm{T}_{\mathrm{MAX}}=$ maximum average temperature; $\mathrm{L}_{\mathrm{V}}=$ linguistic variables; $\mathrm{Q}_{\mathrm{V}}=$ quantitative variables; $\mathrm{BY}=$ biomass yield; $\mathrm{VL}=\mathrm{very}$ low; $\mathrm{L}=$ low; $\mathrm{M}=$ medium; $\mathrm{H}=$ high; $\mathrm{VH}=$ very high; $\mathrm{Hy}$ $=$ hydrogel $\left(\mathrm{kg} \mathrm{ha}^{-1}\right)$

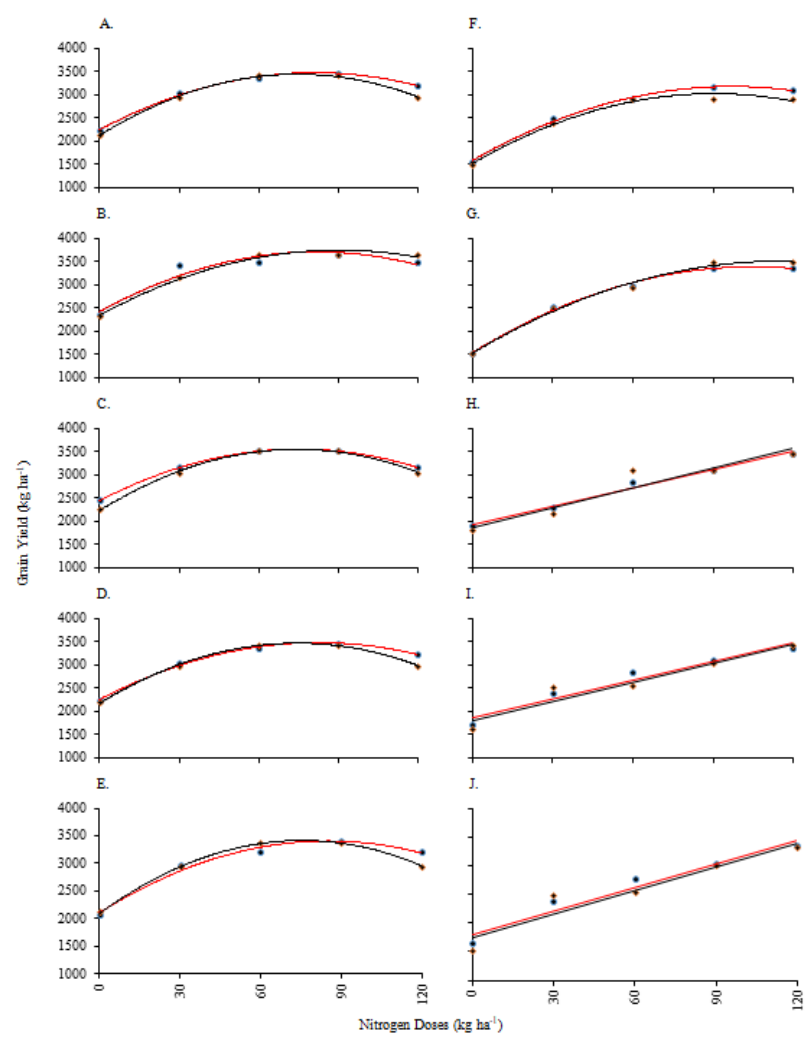

Fig 2. Behavior of grain yield observed and estimated by fuzzy logic. GYo = Observed grain yield $\left(\mathrm{kg}^{-1}\right)$; $\mathrm{GYe}=\mathrm{Estimated}$ grain yield $\left(\mathrm{kg}^{-1}\right)$; $A, B$, $\mathrm{C}, \mathrm{D}, \mathrm{E}=\mathrm{Graph}$ of grain yield in the soybean/oat system under conditions of use of hydrogel $0,30,60,90$ and $120 \mathrm{~kg} \mathrm{ha}^{-1}$, respectively; F, G, H, I, J = Graph of grain yield in the corn/oat system under conditions of use of hydrogel $0,30,60,90$ and $120 \mathrm{~kg}^{-1}$, respectively; $\bullet=$ Observed grain yield; $\bullet$ Grain yield estimated by fuzzy logic; $-G Y_{O A}=-0.186 N^{2}+30.18 N+2241, \mathrm{R}^{2}=99 ;-G Y_{E A}=-0.235 N^{2}+35.18 N+2119, \mathrm{R}^{2}=99 ;-G Y_{O B}=$ $-0.192 N^{2}+31.04 N+2416, R^{2}=93 ;-G Y_{E B}=-0.171 N^{2}+30.97 N+2337, \mathrm{R}^{2}=98 ;-G Y_{O C}=-0.196 N^{2}+29.45 N+2438, \mathrm{R}^{2}=99 ;-G Y_{E C}=$ $-0.236 N^{2}+35.34 N+2226, \mathrm{R}^{2}=99 ;-G Y_{O D}=-0.180 N^{2}+29.72 N+2247, \mathrm{R}^{2}=99 ;-G Y_{E D}=-0.232 N^{2}+34.67 N+2167, \mathrm{R}^{2}=99 ; ;-G Y_{O E}=$ $-0.179 N^{2}+30.47 N+2110, \mathrm{R}^{2}=98 ;-G Y_{E E}=-0.231 N^{2}+34.81 N+2102, \mathrm{R}^{2}=99 ;-G Y_{O F}=-0.169 N^{2}+32.83 N+1577, \mathrm{R}^{2}=99 ;-G Y_{E F}=$ $-0.184 N^{2}+33.35 N+1511, \mathrm{R}^{2}=98 ;-G Y_{O G}=-0.168 N^{2}+35.29 N+1528, \mathrm{R}^{2}=99 ;-G Y_{E G}=-0.148 N^{2}+34.31 N+1518, \mathrm{R}^{2}=99 ;-G Y_{O H}=$ $12.99 N+1928, \mathrm{R}^{2}=98 ;-G Y_{E H}=14.1 N+1862, \mathrm{R}^{2}=90 ;-G Y_{O I}=13.53 N+1859, \mathrm{R}^{2}=95 ;-G Y_{E I}=13.76 N+1796, \mathrm{R}^{2}=92 ;-G Y_{O J}=13.6 N+$ $1809, \mathrm{R}^{2}=95 ;-G Y_{E J}=13.7 N+1750, \mathrm{R}^{2}=91 ; \mathrm{R}^{2}=$ coefficient of determination; $N=$ Nitrogen. 
Table 3. Fuzzy logic in the simulation of oat yield by nitrogen and temperature in the use of the hydrogel, in the soybean/oat system.

\begin{tabular}{|c|c|c|c|c|c|c|c|c|c|c|}
\hline \multirow{2}{*}{$\frac{\mathrm{N}}{\left(\mathrm{kg} \mathrm{ha}^{-1}\right)}$} & \multicolumn{3}{|c|}{$\mathrm{GY}\left(\mathrm{kg} \mathrm{ha}^{-1}\right)$} & \multicolumn{3}{|c|}{ BY $\left(\mathrm{kg} \mathrm{ha}^{-1}\right)$} & \multicolumn{2}{|c|}{ Fuzzy $\left(\mathrm{kg} \mathrm{ha}^{-1}\right)$} & \multicolumn{2}{|c|}{$\operatorname{ror}\left(\mathrm{kg} \mathrm{ha}^{-1}\right)$} \\
\hline & $\mathrm{L}_{1}$ & $\dot{X}$ & $U_{1}$ & $L_{1}$ & $\dot{X}$ & $\mathrm{U}_{1}$ & GY & $\mathrm{BY}$ & GY & BY \\
\hline \multicolumn{11}{|c|}{ Joint Analysis (2014+2015) } \\
\hline 0 & 1987 & $2220 c$ & 2420 & 5067 & $7481 \mathrm{~d}$ & 9553 & 2130 & 8430 & 90 & 949 \\
\hline 30 & 2750 & $3035 \mathrm{~b}$ & 3278 & 8847 & $10414 \mathrm{c}$ & 11758 & 2940 & 9910 & 95 & 504 \\
\hline 60 & 2872 & $3342 \mathrm{a}$ & 3745 & 10854 & $11531 b$ & 12113 & 3390 & 11300 & 48 & 231 \\
\hline 90 & 2934 & 3449 a & 3891 & 11457 & 12466 a & 13332 & 3390 & 12900 & 59 & 434 \\
\hline 120 & 2643 & $3192 b$ & 3663 & 11920 & $13004 \mathrm{a}$ & 14671 & 2940 & 12900 & 252 & 104 \\
\hline Average & 2637 & 3047 & 3399 & 9626 & 11059 & 12285 & 2958 & 11088 & 89 & 29 \\
\hline \multicolumn{11}{|c|}{ Hydrogel $\left(30 \mathrm{~kg} \mathrm{ha}^{-1}\right)$} \\
\hline 0 & 2150 & $2327 d$ & 2478 & 6120 & 8616 e & 10757 & 2310 & 9000 & 17 & 384 \\
\hline 30 & 2892 & $3106 \mathrm{c}$ & 3290 & 8976 & $10613 d$ & 12017 & 3150 & 10300 & 44 & 313 \\
\hline 60 & 2964 & 3483 a & 3929 & 10101 & $11789 \mathrm{c}$ & 13237 & 3630 & 11500 & 147 & 289 \\
\hline 90 & 3170 & $3634 a$ & 4031 & 12164 & 12974 b & 13669 & 3630 & 12900 & 4 & 74 \\
\hline 120 & 3061 & 3466 a & 3814 & 13589 & 14159 a & 14648 & 3630 & 14000 & 164 & 159 \\
\hline Average & 2847 & 3203 & 3508 & 10190 & 11630 & 12648 & 3270 & 11540 & 67 & 90 \\
\hline \multicolumn{11}{|c|}{ Hydrogel $\left(60 \mathrm{~kg} \mathrm{ha}^{-1}\right)$} \\
\hline 0 & 2035 & 2442 c & 2790 & 6344 & $7908 d$ & 9249 & 2240 & 8680 & 202 & 772 \\
\hline 30 & 2783 & $3138 \mathrm{~b}$ & 3442 & 9261 & $9676 c$ & 10031 & 3040 & 9690 & 98 & 14 \\
\hline 60 & 2954 & 3502 a & 3973 & 10514 & $11784 \mathrm{~b}$ & 12873 & 3500 & 10700 & 2 & 1084 \\
\hline 90 & 2985 & $3505 a$ & 3952 & 11400 & $12194 \mathrm{a}$ & 12875 & 3500 & 11800 & 5 & 394 \\
\hline 120 & 2618 & $3146 \mathrm{~b}$ & 3599 & 11680 & $12604 \mathrm{a}$ & 13053 & 3040 & 11800 & 6 & 804 \\
\hline Average & 2675 & 3146 & 3551 & 9839 & 10883 & 11616 & 3064 & 10534 & 82 & 349 \\
\hline \multicolumn{11}{|c|}{ Hydrogel $\left(90 \mathrm{~kg} \mathrm{ha}^{-1}\right)$} \\
\hline 0 & 2041 & $2225 c$ & 2381 & 7156 & $8271 \mathrm{e}$ & 9500 & 2180 & 9460 & 45 & 1189 \\
\hline 30 & 2631 & $3036 \mathrm{~b}$ & 3384 & 9588 & $10112 d$ & 10561 & 2970 & 10500 & 66 & 388 \\
\hline 60 & 2864 & 3335 a & 3740 & 11272 & 11852 c & 12350 & 3420 & 12300 & 85 & 448 \\
\hline 90 & 2963 & 3462 a & 3891 & 12100 & $12669 b$ & 13157 & 3420 & 12600 & 42 & 69 \\
\hline 120 & 2792 & $3222 b$ & 3591 & 12872 & 13485 a & 14012 & 2970 & 13500 & 252 & 15 \\
\hline Average & 2658 & 3056 & 3397 & 10597 & 11277 & 11916 & 2992 & 11672 & 64 & 395 \\
\hline \multicolumn{11}{|c|}{ Hydrogel $\left(120 \mathrm{~kg} \mathrm{ha}^{-1}\right)$} \\
\hline 0 & 1957 & $2070 \mathrm{c}$ & 2168 & 7721 & 8367 e & 8990 & 2110 & 8980 & 40 & 613 \\
\hline 30 & 2488 & $2969 \mathrm{~b}$ & 3381 & 9791 & $10204 d$ & 11300 & 2920 & 11100 & 49 & 896 \\
\hline 60 & 2787 & 3215 a & 3583 & 11660 & 11902 c & 13100 & 3370 & 13000 & 155 & 1098 \\
\hline 90 & 2897 & 3398 a & 3828 & 12359 & $12830 \mathrm{~b}$ & 13234 & 3370 & 13200 & 28 & 370 \\
\hline 120 & 2808 & $3199 \mathrm{~b}$ & 3534 & 12757 & 13759 a & 14617 & 2950 & 14100 & 249 & 341 \\
\hline Average & 2587 & 2970 & 3298 & 10857 & 11412 & 12248 & 2944 & 12076 & 26 & 664 \\
\hline
\end{tabular}

$\mathrm{N}=$ nitrogen; $L_{1}=$ lower limit; $U_{1}=$ upper limit $\left(L_{1}\right.$ and $U_{1}$ of the confidence interval at $5 \%$ probability of error); $\dot{X}=$ average; $G Y=$ grain yield; $B Y=$ biomass yield; Means followed by the same letter within each hydrogel use condition constitute a statistically homogeneous group by the Skott-Knott model with a $5 \%$ probability of error.

Table 4. Fuzzy logic in the simulation of oat yield by nitrogen and temperature in the use of hydrogel in the corn/oat system.

\begin{tabular}{|c|c|c|c|c|c|c|c|c|c|c|}
\hline \multirow{2}{*}{$\begin{array}{l}\mathrm{N} \\
\left(\mathrm{kg} \mathrm{ha}^{-1}\right) \\
\end{array}$} & \multicolumn{3}{|c|}{$\mathrm{GY}\left(\mathrm{kg} \mathrm{ha}^{-1}\right)$} & \multicolumn{3}{|c|}{ BY $\left(\mathrm{kg} \mathrm{ha}^{-1}\right)$} & \multicolumn{2}{|c|}{ Fuzzy $\left(\mathrm{kg} \mathrm{ha}^{-1}\right)$} & \multicolumn{2}{|c|}{$\operatorname{ror}\left(\mathrm{kg} \mathrm{ha}^{-1}\right)$} \\
\hline & $\mathrm{L}_{\mathrm{I}}$ & $\dot{X}$ & $\mathrm{U}_{\mathrm{I}}$ & $\mathrm{L}_{1}$ & $\dot{X}$ & $\mathrm{U}_{\mathrm{I}}$ & GY & BY & GY & BY \\
\hline \multicolumn{11}{|c|}{ Joint Analysis (2014+2015) } \\
\hline \multicolumn{11}{|c|}{ Hydrogel $\left(0 \mathrm{~kg} \mathrm{ha}^{-1}\right)$} \\
\hline 0 & 1240 & $1551 \mathrm{c}$ & 1818 & 4469 & 5993 e & 7299 & 1480 & 6040 & 71 & 47 \\
\hline 30 & 2163 & $2476 b$ & 2745 & 8393 & $9003 \mathrm{~d}$ & 9527 & 2390 & 9420 & 86 & 417 \\
\hline 60 & 2451 & $2902 \mathrm{a}$ & 3289 & 10080 & $10455 \mathrm{c}$ & 10770 & 2900 & 10500 & 2 & 45 \\
\hline 90 & 2789 & 3144 a & 3449 & 10408 & $10927 \mathrm{~b}$ & 11372 & 2900 & 10700 & 244 & 227 \\
\hline 120 & 2778 & 3094 a & 3364 & 10982 & 11645 a & 12215 & 2900 & 11700 & 194 & 55 \\
\hline Average & 2284 & 2633 & 2933 & 8866 & 9604 & 10222 & 2514 & 9672 & 119 & 67 \\
\hline \multicolumn{11}{|c|}{ Hydrogel $\left(30 \mathrm{~kg} \mathrm{ha}^{-1}\right)$} \\
\hline 0 & 1231 & $1502 \mathrm{~d}$ & 1735 & 4275 & 6416 e & 8253 & 1500 & 5870 & 2 & 546 \\
\hline 30 & 2254 & $2511 \mathrm{c}$ & 2731 & 7726 & $9106 d$ & 10289 & 2490 & 8130 & 21 & 976 \\
\hline 60 & 2655 & $2979 \mathrm{~b}$ & 3258 & 8954 & 9777 c & 10482 & 2930 & 9130 & 49 & 647 \\
\hline 90 & 3014 & 3351 a & 3640 & 9609 & $10488 \mathrm{~b}$ & 11241 & 3480 & 10100 & 129 & 388 \\
\hline 120 & 3009 & 3348 a & 3639 & 10378 & 11189 a & 11885 & 3480 & 11300 & 132 & 111 \\
\hline Average & 2432 & 2738 & 3000 & 8188 & 9395 & 10430 & 2776 & 8906 & 38 & 489 \\
\hline \multicolumn{11}{|c|}{ Hydrogel $\left(60 \mathrm{~kg} \mathrm{ha}^{-1}\right)$} \\
\hline 0 & 1584 & $1891 \mathrm{e}$ & 2154 & 6065 & 7991 e & 9643 & 1790 & 8280 & 101 & 289 \\
\hline 30 & 1982 & $2281 \mathrm{~d}$ & 2537 & 6598 & $8218 \mathrm{~d}$ & 9606 & 2150 & 9500 & 131 & 1288 \\
\hline 60 & 2524 & $2837 \mathrm{c}$ & 3105 & 9320 & 9826 c & 10261 & 3080 & 10200 & 243 & 374 \\
\hline 90 & 2852 & $3097 \mathrm{~b}$ & 3307 & 10349 & $11010 \mathrm{~b}$ & 11577 & 3080 & 11300 & 17 & 290 \\
\hline 120 & 3224 & $3432 \mathrm{a}$ & 3610 & 11599 & $12300 \mathrm{a}$ & 12902 & 3440 & 12200 & 8 & 100 \\
\hline Average & 2433 & 2707 & 2942 & 8786 & 9869 & 10797 & 2708 & 10296 & 1 & 427 \\
\hline \multicolumn{11}{|c|}{ Hydrogel $\left(90 \mathrm{~kg} \mathrm{ha}^{-1}\right)$} \\
\hline 0 & 1374 & 1689 e & 1958 & 4912 & 6736 e & 8300 & 1610 & 6510 & 79 & 226 \\
\hline 30 & 2104 & $2392 d$ & 2639 & 6975 & $8259 \mathrm{~d}$ & 9360 & 2510 & 7850 & 118 & 679 \\
\hline 60 & 2491 & $2811 \mathrm{c}$ & 3086 & 9124 & $9644 \mathrm{c}$ & 10091 & 2550 & 9980 & 261 & 336 \\
\hline 90 & 2812 & $3097 \mathrm{~b}$ & 3342 & 10352 & $10937 d$ & 11440 & 3020 & 11100 & 209 & 163 \\
\hline 120 & 3109 & $3367 a$ & 3589 & 11798 & $12308 \mathrm{a}$ & 12745 & 3420 & 12400 & 53 & 92 \\
\hline Average & 2378 & 2671 & 2922 & 8632 & 9576 & 10387 & 2622 & 9568 & 48 & 8 \\
\hline \multicolumn{11}{|c|}{ Hydrogel $\left(120 \mathrm{~kg} \mathrm{ha}^{-1}\right)$} \\
\hline 0 & 1296 & $1634 \mathrm{e}$ & 1924 & 4471 & 6188 e & 7661 & 1530 & 6140 & 104 & 48 \\
\hline 30 & 2006 & $2363 d$ & 2669 & 6199 & $7691 d$ & 8971 & 2480 & 7630 & 117 & 61 \\
\hline 60 & 2425 & $2757 c$ & 3041 & 8949 & $9521 \mathrm{c}$ & 10011 & 2520 & 10000 & 237 & 479 \\
\hline 90 & 2736 & $3033 \mathrm{~b}$ & 3289 & 10477 & $11014 \mathrm{~b}$ & 11475 & 3010 & 11200 & 23 & 186 \\
\hline 120 & 3061 & $3340 \mathrm{a}$ & 3580 & 12074 & $12591 \mathrm{a}$ & 13035 & 3320 & 12600 & 20 & 9 \\
\hline Average & 2304 & 2625 & 2900 & 8434 & 9401 & 10230 & 2572 & 9514 & 53 & 113 \\
\hline
\end{tabular}

$\mathrm{N}=$ nitrogen; $\mathrm{L}_{1}=$ lower limit; $\mathrm{U}_{\mathrm{I}}=$ upper limit $\left(\mathrm{L}_{1}\right.$ and $\mathrm{U}_{\mathrm{I}}$ of the confidence interval at $5 \%$ probability of error); $\dot{X}=$ average; $G Y=$ grain yield; $B Y=$ biomass yield; Means followed by the same letter within each hydrogel use condition constitute a statistically homogeneous group by the Skott-Knott model with a $5 \%$ probability of error. 

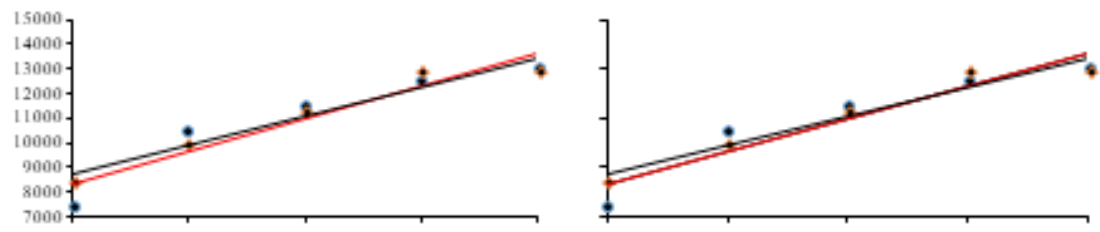

B.

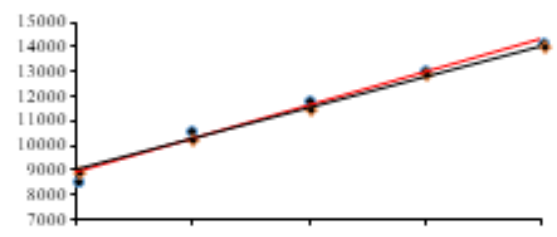

G.
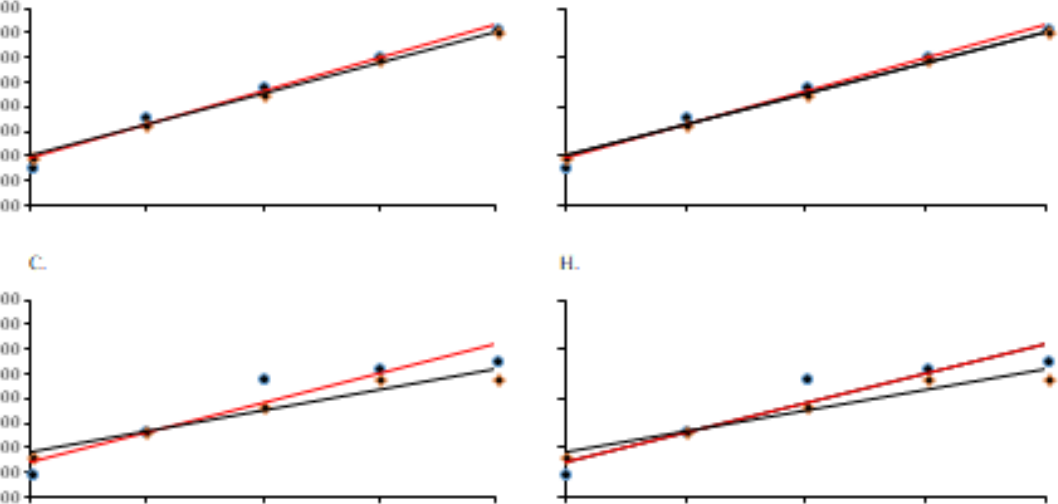

H.
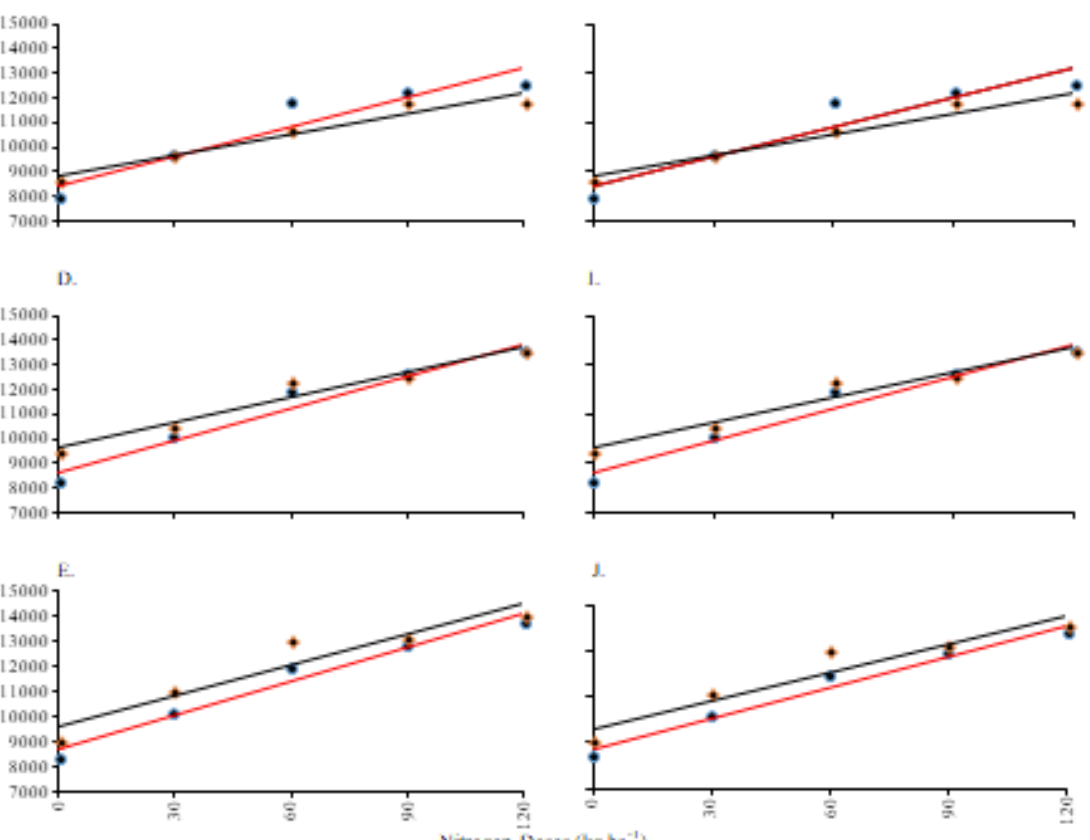

J

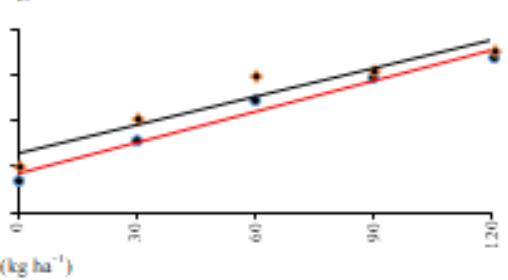

Fig 3. Behavior of biomass yield observed and estimated by fuzzy logic. BYo = Observed biomass yield (kg ha-1); BYe = Estimated biomass yield $\left(\mathrm{kg} \mathrm{ha}^{-1}\right) ; \mathrm{A}, \mathrm{B}, \mathrm{C}, \mathrm{D}, \mathrm{E}=\mathrm{Graph}$ of the biomass yield in the soybean/oat system under conditions of use of hydrogel 0 , $30,60,90$ and $120 \mathrm{~kg} \mathrm{ha}^{-1}$, respectively; F, G, H, I, J = Graph of biomass yield in the corn/oat system under conditions of use of hydrogel 0,30,60,90 and $120 \mathrm{~kg} \mathrm{ha}^{-1}$, respectively; $\bullet=$ Observed biomass yield; $\bullet$ Estimated biomass yield by fuzzy logic. $-B Y_{O A}=$ $43,66 N+8359, \quad \mathrm{R}^{2}=99 ; \quad-B Y_{E A}=3.76 N+8702, \quad \mathrm{R}^{2}=94 ; \quad-B Y_{O B}=44.81 N+8940, \quad \mathrm{R}^{2}=98 ; \quad-B Y_{E B}=42 N+9020, \quad \mathrm{R}^{2}=99 ;$ $-B Y_{O C}=29.45 N+2438, \mathrm{R}^{2}=99 ;-B Y_{E C}=35.34 N+2226, \mathrm{R}^{2}=99 ; ;-B Y_{O D}=29.72 N+2247, \mathrm{R}^{2}=99 ;-B Y_{E D}=34.67 N+$ 2167, $\mathrm{R}^{2}=99 ; ;-B Y_{O E}=30.47 \mathrm{~N}+2110, \mathrm{R}^{2}=98 ;-B Y_{E E}=34.81 \mathrm{~N}+2102, \mathrm{R}^{2}=99 ;-B Y_{O F}=32.83 N+1577, \mathrm{R}^{2}=99 ;-B Y_{E F}=$ $33.35 N+1511, \mathrm{R}^{2}=98 ;-B Y_{O G}=35.29 N+1528, \mathrm{R}^{2}=99 ;-B Y_{E G}=34.31 N+1518, \mathrm{R}^{2}=99 ; ;-B Y_{O H}=12.99 N+1928, \mathrm{R}^{2}=98 ;$ $-B Y_{E H}=14.1 N+1862, \mathrm{R}^{2}=90 ; ;-B Y_{O I}=13.53 N+1859, \mathrm{R}^{2}=95 ;-B Y_{E I}=13.76 \mathrm{~N}+1796, \mathrm{R}^{2}=92 ; ;-B Y_{O J}=13.6 \mathrm{~N}+1809$, $\mathrm{R}^{2}=95 ;-B Y_{E J}=13.7 N+1750, \mathrm{R}^{2}=91 ; \mathrm{R}^{2}=$ coefficient of determination; $N=$ Nitrogen.

expression of biomass yield at the highest nutrient dose, indicating a trend towards linearity. The analysis in paired form in the comparison of the real and simulated values by fuzzy logic in each condition of use of the hydrogel showed similar values in the expression of grain biomass yield. These facts report the effectiveness of the rules base developed for the developmental quality of fuzzy modeling.

In the corn/oat system (Table 4), regardless of the use of the biopolymer hydrogel, in general, the increase of $\mathrm{N}$-fertilizer promoted increased yield of biomass and grains to the highest nutrient dose, indicating a trend of linearity in the more restrictive $\mathrm{N}$-residual condition. In the simulation of grain yield by diffuse logic, the results were close to those obtained by bio-experimentation. Although differences are obtained between the different points of analysis, all presented results within the established confidence interval, independent of the conditions of nitrogen and hydrogel use. In the overall average in each hydrogel use condition, results between the observed and simulated values were very close to the expression of biomass and grain yield. The condition of reduced release of $\mathrm{N}$-residual shows behavior of yield growth with the provision of higher doses of fertilizer. Therefore, a trend of linearity between the real and simulated points by the fuzification process, also ensuring quality of the rules base established for this system. The results obtained from the simulations, although closer to or far from the actual points of the bio-experiment, all the results at their respective levels were within the confidence 
interval of the mean. A condition that justifies the established basis of rules and the possibility of using nebulous logic in the predictability of biomass and oat grain yields involving nonlinearity of air temperature with important management technologies.

The fuzzy models are techniques that allow the description of complex systems, produced from rules, that must be elaborated by specialists, providing their experience to the elaboration of an inference system (Freitas et al., 2013). In this perspective, fuzzy logic has been increasingly used in different areas of knowledge, allowing to assign linear and non-linear effects of the processes with the experience gained from the observer (Malaman \& Amorim, 2017). In agriculture Silva et al. (2014) made use of the fuzzy neuro in the estimation of grain yield of wheat as a function of nitrogen fertilization. Francisco et al. (2007), developed a fuzzy model to estimate soybean area from vegetation indexes of images, being highly correlated with estimates recorded by official bodies. Giusti \& Marsili-Libelli, (2015), studied the use of fuzzy logic as irrigation controller by the cumulative days, total water applied to the crop and evapotranspiration in corn, kiwi and potato crops, being highly comparative to existing irrigation agricultural models.

\section{Materials and methods}

\section{Crop area description}

The works were developed in the field, in the agricultural years of 2014 and 2015, in the municipality of Augusto Pestana, RS, Brazil (28 $28^{\circ} 26^{\prime} 30^{\prime}$ 'S and $54^{\circ} 00^{\prime} 58$ " W longitude). The soil of the experimental area was classified as Typical Dystrophic Red Latosol (Oxisol) and the climate of the region, by classification of Köppen, Cfa type (subtropical humid). In the study, ten days before sowing, soil analysis was performed and the following chemical characteristics were identified (Tedesco et al., 1995): i) maize/oat system $\left(\mathrm{pH}=6,5 ; \mathrm{P}=34,4 \mathrm{mg} \mathrm{dm}^{-3} ; \mathrm{K}=262 \mathrm{mg} \mathrm{dm}^{-3} ; \mathrm{OM}=2,9 \% ; \mathrm{Al}=\right.$ $0 \mathrm{cmol}_{\mathrm{c}} \mathrm{dm}^{-3} ; \mathrm{Ca}=6,6 \mathrm{cmol}_{\mathrm{c}} \mathrm{dm}^{-3}$ and $\mathrm{Mg}=3,4 \mathrm{cmol}_{\mathrm{c}} \mathrm{dm}^{-3}$ ) and; ii) soybean/oat system $\left(\mathrm{pH}=6,2 ; \mathrm{P}=33,9 \mathrm{mg} \mathrm{dm}^{-3} ; \mathrm{K}=\right.$ $200 \mathrm{mg} \mathrm{dm}^{-3} ; \mathrm{OM}=3,0 \%$; $\mathrm{Al}=0 \mathrm{cmol}_{\mathrm{c}} \mathrm{dm}^{-3} ; \mathrm{Ca}=6,5 \mathrm{cmol}_{\mathrm{c}}$ $\mathrm{dm}^{-3}$ and $\left.\mathrm{Mg}=2,5 \mathrm{cmol}_{\mathrm{c}} \mathrm{dm}^{-3}\right)$. Independent of the agricultural year, sowing was carried out in the third week of June, according to the cultivation recommendation, considering two succession systems of high and low release of residual $\mathrm{N}$, corn/oat and soybean/oat systems, respectively.

\section{Experimental design}

At sowing, the seeder was used in the composition of the plot with 5 lines of $5 \mathrm{~m}$ in length and spacing between rows of $0.20 \mathrm{~m}$, forming the experimental unit of $5 \mathrm{~m}^{2}$. The population density was 400 viable seeds $\mathrm{m}^{-2}$, using the URSCorona white oat cultivar. During the execution of the study, tebuconazole fungicide of commercial name FOLICUR ${ }^{\circ}$ CE was applied at a dosage of $0.75 \mathrm{~L} \mathrm{ha}^{-1}$. Weed control was carried out with commercially available metysulfuronmethyl herbicide (ALY) at the dose of $4 \mathrm{~g} \mathrm{ha}^{-1}$ of the commercial product and additional weeding when necessary. In the experiments, at sowing, 45 and $30 \mathrm{~kg} \mathrm{ha}^{-1}$ of $\mathrm{P}_{2} \mathrm{O}_{5}$ and $\mathrm{K}_{2} \mathrm{O}$ were applied, based on the levels of $\mathrm{P}$ and $\mathrm{K}$ in the soil for expectation of grain yield of $3 \mathrm{t} \mathrm{ha} a^{-1}$, respectively, and $10 \mathrm{~kg} \mathrm{ha}^{-1}$ of nitrogen (except in the standard experimental unit), with the remainder to contemplate the doses proposed for top dressing at the fourth expanded leaf stage. It should be noted that the different doses of the biopolymer hydrogel were added to the oat seed, being in the soil at the same depth and line of cultivation, approximately $3 \mathrm{~cm}$ deep. It is noteworthy that the hydrogel is a fine granulated biopolymer, which when in contact with moisture forms a gelatinous structure, capable of storing water and making it available to plants for a long period of time.

In each succession system of high and low residual $\mathrm{N}$ release (corn/oat and soy/oat systems) two experiments were conducted, one to quantify biomass yield (BY, $\mathrm{kg} \mathrm{ha}^{-1}$ ), the other to estimate grain yield (GY, $\left.\mathrm{kg} \mathrm{ha}^{-1}\right)$. Therefore, in the four experiments, the experimental design was a randomized block with four replicates, with the hydrogel and nitrogen treatments, following a $5 \times 5$ factorial scheme in the sources of variation of hydrogel doses at levels 0,30 , 60,90 and $120 \mathrm{~kg} \mathrm{ha}^{-1}$, and doses of $\mathrm{N}$-fertilizer (Source Urea) at levels $0,30,60,90$ and $120 \mathrm{~kg} \mathrm{ha}^{-1}$.

\section{Data measurement}

The grain yield was obtained by cutting three central lines of each plot at the maturity stage, with grain moisture around $22 \%$. The plants were harvested with a stationary harvester and directed to the laboratory for correction of grain moisture to $13 \%$ and weighing to estimate grain yield (GY, kg $\left.h \mathrm{a}^{-1}\right)$. In the experiments aimed at quantifying the biomass yield, the plant material was harvested close to the soil, starting from the collection of a linear meter of the three central lines of each plot, at 120 days after emergence. The biomass samples were directed to a forced air oven at $65{ }^{\circ} \mathrm{C}$ until constant weight was reached to estimate the total biological yield (BY, $\mathrm{kg} \mathrm{ha}^{-1}$ ).

\section{Modeling for Fuzzy Logic}

Based on the initial analysis of the data, the rules were developed, in linguistic terms, with the help of a plant specialist. The input variables of the model (independent variables) were nitrogen $(\mathrm{N})$ and maximum air temperature $\left(T_{M A ́ X}\right)$. It is noteworthy that the maximum air temperature value was obtained by the Automatic Weather Station, installed at $\mathbf{2 0 0}$ meters distance from the experiment. The output variables (dependent variables) were grain yield (GY) and biological productivity (BY). For the $\mathrm{T}_{\text {MÁx }}$ independent variable $(\stackrel{\circ}{ })$, the domain in the range 21 to 25 was considered, representing the bands: $<22.5^{\circ} \mathrm{C}$ (low temperature) and $>22.5 \circ \mathrm{C}$ (high temperature). For the independent variable $\mathrm{N}\left(\mathrm{kg} \mathrm{ha}^{-1}\right)$, the range zero to 120 was considered, representing the intervals: $<15$ [very low dose (VL)]; 15-45 [low dose (L)]; 45-75 [mean dose (M)]; 75-105 [high dose $(\mathrm{H})$ ] and $>105$ [very high dose $(\mathrm{VH})$ ]. For the output variable (dependent variable), the image intervals were the maximum and minimum values of the experimentally collected biomass and oat grain yield data, considering the cumulative effect of the growing years, in each hydrogel use condition. Thus, in the development of the modeling process 10 simulators were constructed. The variable GY was divided into four equidistant intervals (Table 1 ), being: low $(\mathrm{L})$, medium $(\mathrm{M})$, high $(\mathrm{H})$ and very high $(\mathrm{VH})$ and $\mathrm{BY}$ variable in five intervals (Table 2 ): very low $(\mathrm{VL})$, low $(\mathrm{L})$, medium $(\mathrm{M})$, high $(\mathrm{H})$ and very high $(\mathrm{VH})$. For the simulations the nitrogen doses and the average maximum air temperature in the years of cultivation were used as inputs. 
In Tables 1 and 2, the classifications are presented for the simulation of grain and biomass yield, respectively, as a function of nitrogen ( $\mathrm{kg} \mathrm{ha}^{-1}$ ) and maximum air temperature $(\stackrel{\circ}{ })$, respectivel in each hydrogel condition.

In the development of fuzzy logic programming, the Fuzzy Logic Toolbox of MATLAB ${ }^{\circledR}$ software was used. In the prediction applications the Mamdani fuzzy model was used, which includes interface modules in the transformation of input variables into fuzzy sets and, later, outputs in proportional numerical quantities (Cecconello et al., 2010). The fuzzification process was carried out by four successive modules. In module 1 (fuzzification), the information of the input variables was mathematically modeled by means of fuzzy sets. From the expert, for each entry variable, linguistic terms representing the states of this variable were assigned and, for each linguistic term, a fuzzy set associated with a pertinence function. In module 2 (rule base), the variables were adjusted in their linguistic classifications, where each rule base satisfied the following structure:

If $\mathrm{A}$ is in $A_{i}$ then $B$ is in $B_{i}$

where $A_{i}$ and $B_{i}$ are the fuzzy sets. The expression $A$ is in $A_{i}$ means that $\mu_{A_{i}}(a) \in[0,1]$. Both the $\mathrm{A}_{\mathrm{i}}$ and $\mathrm{B}_{\mathrm{i}}$ sets are the Cartesian product of fuzzy sets, that is, $A_{i}=A_{i 1} \times A_{i 2} \times$ $\ldots \times A_{\mathfrak{J}}$ e $B_{i}=B_{i 1} \times B_{i 2} \times \ldots \times B$. In this case, each set fuzzy $A_{i j}$ and $B_{i k}$ represented a linguistic term for the $\mathrm{j}^{\text {th }}$ input variable and $\mathrm{k}^{\text {th }}$ output variable, and the expression $A$ is in $A_{i}$ which means:

$\mu_{A_{i}}(a)=\min \left\{\mu_{A_{i 1(a)}}, \mu_{A_{i 2(a)}}, \ldots, \mu_{A_{\Im}(a)}\right\} \in[0,1]$

(1)

In module 3 (inference), the logical connectives used to establish the fuzzy relationship for modeling the rule base were defined. The relationship between the linguistic variables was characterized by the operator (MIN) of the fuzzy system. In each rule we considered a fuzzy relation $R_{i}$ with degree of pertinence for each pair $(a, b)$ :

$\mu_{R_{i}}(a, b)=\min \left\{\mu_{A_{i}}(a), \mu_{B_{i}}(b)\right.$

The relationship between each rule is characterized by the operator (MAX), of the fuzzy relation $R$ that represents the model determined by a rule base obtained by the MAX union of each individual rule, so that for each pair $(a, b)$ is obtained:

$\mu_{R}(a, b)=\max _{1 \leq i \leq n}\left\{\mu_{A_{i}}(a)_{B_{i}}^{\mu}(b)\right\}$

where ${ }^{\wedge}$ represents the MIN operator.

By Mamdani's method the pertinence function of $B$ is given by:

$\left.\mu_{B}(b)=\max _{1 \leq i \leq n}\left\{\max _{a} \mu_{A}(a)_{A i}^{\mu}(a)\right\}_{B i}^{\mu}(b)\right\}$

If the input is a unitary classical set, then $\mu_{A}(a)=1 \mathrm{e}$ $\mu_{A i}(a) \leq 1$. Thus, the above expression results in:

$\mu_{B}(b)=\max _{1 \leq i \leq n}\left\{\mu_{A i}(a)_{B i}^{\mu}(b)\right\}$

Therefore, the fuzzy set $B$ represents the action for each input $A$.

In module 4 (defuzzification), the state of the fuzzy output variable gives the numerical value. One of the main methods of defuzzification is the center of mass for continuous variables, given by the expression:

$m(B)=\frac{\int b_{\mu B}(b) d b}{\int \mu_{B}(b) d b}$

and of discrete variables by the expression:

$m(B)=\frac{\sum_{b} b_{\mu B}(b) d b}{\sum_{b} b_{\mu B}(b) b}$

The fuzzy controller is described as a function $f: R^{n} \rightarrow R^{m}$, since given an input value, there is a single corresponding output value.

For validation of the rules base and the simulated values of biomass and oat grain yields by fuzzy logic, the polynomial regression behavior and parameters obtained from bioexperimentation under real growing conditions were considered. And of the lower and upper limits of the confidence interval of the average values of two agricultural crops, at a level of $5 \%$ of error probability. In the determination of the regression models and confidence interval, the Genes computational program was used.

\section{Conclusions}

The pertinence functions and the linguistic values established in the input and output variables to simulate the biomass and oat grain yield in the succession systems are adequate.

The fuzzy model makes it possible to estimate efficiently the biomass and oat grain yield under the conditions of use of the hydrogel as a function of the nitrogen doses and maximum air temperature, adding to the existing models of simulation.

\section{Acknowledgment}

To the CAPES, CNPq, FAPERGS, the UNIJUI and to IFRS for the financial support to the research and for the Scientific and Technological Initiation Scholarship, Postgraduate Scholarship and Research Productivity Grant. To the Graduate Program in Mathematical Modeling at UNIJUí for the resources available in the development of this research, which was part of the master's thesis of the first author of this work.

\section{References}

Acosta JÁ de A, Amado TJC, Silva LSda, Santi A, Weber MA (2014) Decomposição da fitomassa de plantas de cobertura e liberação de nitrogênio em função da quantidade de resíduos aportada ao solo sob sistema plantio direto. Ciência Rural. 44(5):801-809.

Arenhardt EG, Silva JAG Da, Arenhardt LG, Carbonari HP, de Oliveira AC (2017) The Nitrogen In Grain Yield And At Lodging Oat Cultivars. International Journal of Current Research. 9(01):45564-45571.

Arenhardt EG, Silva JAGDa, Gewehr E, Costa De Oliveira A, Binelo MO, Valdiero A, De CLAR (2015) The nitrogen supply in wheat cultivation dependent on weather conditions and succession system in southern Brazil. African Journal of Agricultural Research. 10(48):43224330.

Brezolin AP, Gonzalez Da Silva JA, Roos-Frantz F, Binelo MO, Menegassi CA, Krüger, Dornelles EF (2017) Wheat yield obtained from nitrogen dose and fractionation. African Journal of Agricultural Research. 12(8):566-576.

Castro GSA, Costa CHM, Neti JF (2012) Ecofisiologia da aveia branca. Scientia Agraria Paranaensis. 11(3):1-15.

Cecconello MS, Silva JDM, Bassanezi R (2010) Sistemas Dinâmicos Fuzzy: Modelagens Alternativas Para Sistemas Biológicos. Sociedade Brasileira de Matemática Aplicada e Computacional (Vol. 50).

Crestani M, Rosa H, Barbieri L, Gonzalez JA, Silva D, Irajá F, Costa De Oliveira, A (2014). Importância e Dinâmica de Caracteres na Aveia Produtora de Grãos. Embrapa Clima Temperado. Documentos. 376:1-59.

De S, Silva A, Lima JSS (2009) Lógica fuzzy no mapeamento de variáveis indicadoras de fertilidade do solo fuzzy logic to map of variables indicative of soil fertility. Idesia (Arica), 27(3):41-46. 
Francisco J, Antunes G, Zullo Júnior J (2007) Aplicação de lógica fuzzy para estimativa de área plantada da cultura de soja utilizando imagens AVHRR-NOAA. Master, Unicamp, 35-42. Recuperado de

Freitas MC, De Peixoto M da S, Vieira JGV (2013) Uma abordagem fuzzy para análise de decisão na distribuição de leite Tipo A. Rev. Inst. Laticínios Cândido Tostes. 68(395):15-24.

Giusti E, Marsili-Libelli S (2015) A Fuzzy Decision Support System for irrigation and water conservation in agriculture. Environmental Modelling \& Software. 63: 7386.

Guarienti EM, Ciacco CF, Rocca G, Jesus L De, Del A, Maria C, Camargo DO (2004) Influência das temperaturas mínima e máxima em características de qualidade industrial e em rendimento de grãos de trigo. Ciênc Tecnol Aliment. 24(4): 505-515.

Guilherme MR, Aouada FA, Fajardo AR, Martins AF, Paulino AT, Davi MT, Muniz EC (2015) Superabsorbent hydrogels based on polysaccharides for application in agriculture as soil conditioner and nutrient carrier: A review. European Polymer Journal. 72:365-385.

Hideo C, Da Costa M, Alexandre C, Crusciol C, Soratto RP, Neto JF, Moro E (2016) Nitrogen fertilization on palisadegrass: phytomass decomposition and nutrients release. Agropec Trop. 46(2):159-168.

Leal AJF, Miguel EP, Baio FHR, Neves D de C, Leal UAS (2015) Redes neurais artificiais na predição da produtividade de milho e definição de sítios de manejo diferenciado por meio de atributos do solo. Bragantia. 74(4): 436-444.

Malaman CS, Amorim A (2017) Método Para Determinação De Valores Na Avaliação Imobiliária: comparação entre o modelo de regressão linear e lógica fuzzy. BCG - Boletim de Ciências Geodésicas, 23(1):87-100.

Mantai RD, Silva JAG da, Sausen ATR, Costa JSP, Fernandes SBV, Ubessi C (2015) A eficiência na produção de biomassa e grãos de aveia pelo uso do nitrogênio. Revista Brasileira de Engenharia Agrícola e Ambiental. 19:343-349.

Marolli A, da Silva JAG, Sawicki S, Binelo MO, Scremin AH, Reginatto DC, Dornelles EF, Lambrecht DM (2018) A simulação da biomassa de aveia por elementos climáticos, nitrogênio e regulador de crescimento. Arquivo Brasileiro de Medicina Veterinária e Zootecnia. 70(2):535-544.

Marolli A, Da Silva JAG, Romitti MV, Mantai RD, Hawerroth MC, Scremin OB (2017) Biomass and grain yield of oats by growth regulator. Revista Brasileira de Engenharia Agrícola e Ambiental, 2121(33):163-168.
Marolli A, Silva JÁ, Da Romitti MV, Mantai RD, Scremin OB, Frantz RZ, Lima ARC De (2017) Contributive effect of growth regulator Trinexapac-Ethyl to oats yield in Brazil. African Journal of Agricultural Research. 12(10):795-804.

Mota, GLA, Feitosa, RQ, Coutinho, HLC, Liedtke, CE, Müller, S, Pakzad, K, Meirelles, MSP (2007) Multitemporal fuzzy classification model based on class transition possibilities. https://doi.org/10.1016/j.isprsjprs.2007.04.001

Neto JF, Crusciol CAC. Soratto RP, da Costa CHM (2012) Consórcio de guandu-anão com milheto: Persistência e liberação de macronutrientes e silício da fitomassa. Bragantia. 71(2):264-272.

Oliveira RA, Rezende LS, Martinez MA, Miranda GV (2004) Influência de um polímero hidroabsorvente sobre a retenção de água no solo. Revista Brasileira de Engenharia Agrícola e Ambiental. 8(1):160-163.

Reza H, Moghadam T, Zahedi H, Ghooshchi F (2011) Oil quality of canola cultivars in response to water stress and super absorbent polymer application. Agropec Trop. 41(4): 579-586.

Sartore L, Vox G, Schettini E (2013) Preparation and Performance of Novel Biodegradable Polymeric Materials Based on Hydrolyzed Proteins for Agricultural Application. Journal of Polymers and the Environment. 21(3):718-725.

Schiassi L, Yanagi Júnior T, Reis GM, Abreu LHP, Campos AT, Castro J de O (2015) Modelagem Fuzzy aplicada na avaliação do desempenho de frangos de corte. Revista Brasileira de Engenharia Agrícola e Ambiental. (February), 140-146.

Silva JAG, Neto CJG, Fernandes SBV, Mantai RD, Scremin OB, Pretto R (2016) Revista Brasileira de Engenharia Agrícola e Ambiental Nitrogen efficiency in oats on grain yield with stability A eficiência do nitrogênio em aveia na produtividade de grãos com estabilidade. Revista Brasileira de Engenharia Agrícola e Ambiental. 20(12):1095-1100.

Silva AAV, Silva IAF, Filho MCMT, Buzetti S, Teixeira MCM (2014) Estimativa da produtividade de trigo em função da adubação nitrogenada utilizando modelagem neuro fuzzy. Revista Brasileira de Engenharia Agícola e Ambiental. 18(2):1-6.

Tedesco MJ, Gianello C, Bissani CA, Bohnen H, Volkweiss SJ (1995) Análise de solo, plantas e outros materiais. 2.ed. Porto Alegre: UFRGS. 174p

Ward PS, Bell AR, Droppelmann K, Benton TG (2018) Land Use Policy Early adoption of conservation agriculture practices: Understanding partial compliance in programs with multiple adoption decisions. Land Use Policy, 70(October 2017), 27-37. 